\title{
Efficacy and safety of systemic isotretinoin treatment for moderate to severe acne (insights from the real-life clinical setting)
}

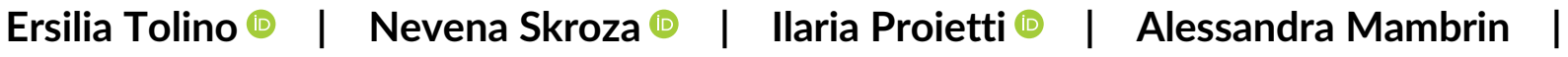 Veronica Balduzzi | Anna Marchesiello ( ) | Patrizia Maddalena |

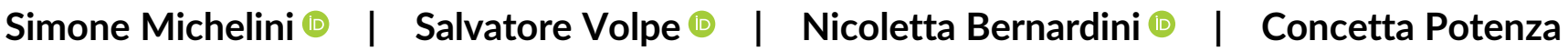

Dermatology Unit "Daniele Innocenzi", Department of Medical-Surgical Sciences and Biotechnologies, Sapienza University of Rome, Polo Pontino, Terracina, Italy

Correspondence

Ersilia Tolino, Dermatology Unit "Daniele Innocenzi," "A. Fiorini" Hospital, Via Firenze, 1, 04019, Terracina (LT), Italy.

Email: ersiliatolino@gmail.com

\begin{abstract}
Acne is a chronic inflammatory relapsing disease that affect predominantly adolescents, with scarring as a frequent sequele. Early and appropriate therapy allows better management of the disease, longer remission, scars risk reduction, and improvement of quality of life. According to therapeutic algorithm, systemic isotretinoin can be used in severe acne and also in moderate forms resistant to other systemic treatments. The aims of this real-life observational study were to determine and compare the effectiveness of isotretinoin evaluated by Global Acne Grading System and Acne Quality of Life in moderate and in severe acne, correlation between efficacy and cumulative dose of isotretinoin, tolerability, and recurrence rate. Moreover, the differences in efficacy and tolerability between male and female patients were compared. The treatment with systemic isotretinoin led to an improvement in acne severity and quality of life in all observed subjects.
\end{abstract}

\section{KEYWORDS}

acne, antibiotics, isotretinoin, moderate acne, severe acne, systemic therapy

\section{$1 \mid$ INTRODUCTION}

Acne is a common chronic inflammatory multifactorial skin disease involving pilosebaceous unit. It affects $80 \%-90 \%$ of adolescents of both sexes even if it can occur at any age. Acne may be classified as mild, moderate or severe based on the number and clinical type of skin lesions. ${ }^{1,2}$

Acne therapy is based on use of topical or systemic agents, depending on clinical severity and psychosocial bourden. ${ }^{3}$ Lesions clearance, scars prevention, and improvement of quality of life are the main goals of therapy.

The use of systemic isotretinoin ${ }^{3}$ in severe acne and in moderate acne resistant to other systemic treatments demonstrated high efficacy, longstanding remission, as well as prevention and reduction of scars. ${ }^{4}$
However, some authors suggested its use also as first-line therapy for moderate acne due to its high effectiveness. ${ }^{5}$

Isotretinoin is a synthetic analogue of vitamin A, which acts inhibiting the differentiation of sebocytes and sebaceous glands function and promotes regeneration and skin repair.

Prevention of scars and durable improvement of the quality of life are considered to be of paramount importance. This position states that concerns about adverse events (AEs) and teratogenic risk should be managed and should not hinder the prevention of acne sequelae. Attentive management of birth control and exclusion of patients with a suicide-prone profile seem to provide a safe use of isotretinoin. ${ }^{6-10}$ As a debate on this subject is still present in literature, the balance of benefits and drawbacks of isotretinoin in real life is to be better understood. 
We conducted an observational real-life monocentric study on patients with moderate to severe acne, treated with oral isotretinoin in order to investigate its effectiveness and safety.

\section{2 | PATIENTS AND METHODS}

Patients with severe acne or moderate acne resistant to other systemic treatments who had received oral isotretinoin, were enrolled in this study. All female patients received oral contraceptive treatment according to current guidelines. ${ }^{6}$

\subsection{Assessments}

Clinical severity of acne was evaluated by the Global Acne Grading System (GAGS; moderate acne was scored 19-30, severe acne was scored $>30$ ) while quality of life was measured by Assessment of Quality of Life (AQoL) questionaire at baseline and at the end of treatment. ${ }^{11-13}$

All AEs during treatment period were recorded.

Primary endpoints were: clinical improvement (reduction of GAGS score) and advanced quality of life (increase of AQoL value) at the end of first treatment cycle, efficacy related with the cumulative dose of isotretinoin, tolerability, and possible recurrence rate. As secondary endpoints, differences in efficacy and tolerability were compared in male and female patients.

\section{3 | RESULTS}

A total of 100 patients (59 males and 41 females) were enrolled: 32 patients with nodulocystic and 68 with papulopustular acne. Moderate acne was observed in 64 patients (59\% of males and $71 \%$ of females) while 36 presented with severe acne ( $41 \%$ of males and $29 \%$ of females). The mean age at the beginning of oral isotretinoin therapy was 22.2 years (range 16-40): 19.4 in males (range 16-33) and 26.5 in females (range 17-40).

Patients received 1 or at most 3 cycles of oral isotretinoin; where the mean duration of the first cycle was 7.68 months.

Daily administered mean dose was $0.41 \mathrm{mg} / \mathrm{kg}$ (range $0.2-0.6 \mathrm{mg} / \mathrm{kg} / \mathrm{day}$ ) in the overall population, with almost equal gender distribution: $0.40 \mathrm{mg} / \mathrm{kg} /$ day (range $0.2-0.6 \mathrm{mg} / \mathrm{kg} / \mathrm{day}$ ) for male patients and $0.43 \mathrm{mg} / \mathrm{kg} /$ day (range $0.3-0.6 \mathrm{mg} / \mathrm{kg} /$ day) for females. Mean cumulative dose was $94.94 \mathrm{mg} / \mathrm{kg}(36-180 \mathrm{mg} / \mathrm{kg})$ in the overall population: $93.51 \mathrm{mg} / \mathrm{kg}$ (range $36-162 \mathrm{mg} / \mathrm{kg} /$ day) in males and $97.04 \mathrm{mg} / \mathrm{kg}$ (range $54-180 \mathrm{mg} / \mathrm{kg}$ ) in females.

\section{$3.1 \mid$ Efficacy}

Baseline GAGS mean score was 27 that was reduced to 3 at the end of treatment. Mean AQoL score was 60 at baseline and 90 after treatment (Table 1).
TAB LE 1 GAGS and AQoL values at baseline and after treatment and improvement percentages

\begin{tabular}{llcc} 
Variables & Total T0 & Total T1 & \% improvement \\
\hline GAGS & $27(9-38)$ & $3(0-16)$ & $89 \%$ \\
\hline AQoL & $60(14-95)$ & $90(40-110)$ & $33 \%$ \\
\hline
\end{tabular}

TABLE 2 GAGS and AQoL improvement percentage in relation with acne severity

\begin{tabular}{|lll|}
\hline Variables & Moderate acne & Severe acne \\
\hline GAGS \% improvement & $89 \%$ & $60 \%$ \\
\hline AQoL \% improvement & $37 \%$ & $23 \%$ \\
\hline
\end{tabular}

TABLE 3 GAGS and AQoL improvement percentage in relation with isotretinoin cumulative dose

\begin{tabular}{|lllll|}
\hline Variables & Low & Med-low & Med-high & High \\
\hline GAGS \% improvement & $65 \%$ & $85 \%$ & $92 \%$ & $93 \%$ \\
\hline AQoL \% improvement & $21 \%$ & $30 \%$ & $33 \%$ & $35 \%$ \\
\hline
\end{tabular}

No differences in treatment response, according to gender, were found in GAGS and in AQoL. Efficacy of isotretinoin was evaluated according to acne severity: improvement of GAGS score was $89 \%$ in moderate acne and $60 \%$ in severe acne; improvement of Acne-QoL score was $37 \%$ in moderate acne and $23 \%$ in severe acne (Table 2).

Furthermore efficacy of isotretinoin was evaluated in four subgroups of patients according to the cumulative dose (low $<80 \mathrm{mg} / \mathrm{kg}$; low-medium = 80-100 mg/kg; medium-high = 100-120 mg/kg; high $>120 \mathrm{mg} / \mathrm{kg}$ ). Daily isotretinoin dose was gradually tapered in order to obtain a maximum tolerability for each patient and to increase contemporarily the safety and efficacy of the drug. This is the reason why the overall cumulative dose in all subgroups was lower compared with dosage from data sheet. Improvement of GAGS score was 65\% in the low-dose group, $85 \%$ in the low-medium-dose group, $92 \%$ in patients receiving medium-high-dose, and $93 \%$ in the high-dose group. Accordingly, the improvement of quality of life score was $21 \%$ in the group receiving a low cumulative dose, $30 \%$ in a low-medium dose, $33 \%$ in a medium-high dose and $35 \%$ in a high cumulative dose group (Table 3).

Only $9 \%$ of patients presented acne recurrence after the end of treatment with isotretinoin during the first year of follow up. However females demonstrated higher percentage (12\%) than males (6\%). In relation to the dosage, recurrence distribution by gender was as follows: $20 \%$ in males and $25 \%$ in females treated with a low dose, $15 \%$ of males and $10 \%$ of females with a low-medium dose, $5 \%$ of males, and $15 \%$ of females with a medium-high dose. No recurrences were observed in patients who received a high cumulative dose. Related to severity, percentage of recurrence was $11 \%$ in moderate acne and $30 \%$ in severe acne. 


\section{2 | Tolerability}

A total of $80 \%$ of patients reported $\geq 1 \mathrm{AE}$. From those, $87 \%$ reported skin xerosis and cheilitis, while the others reported at least one of AEs: epistaxis, headache, myalgia, and blurring of vision. Haematochemical changes, presented mainly as slight increase in transaminases and cholesterolemia, were observed in $9 \%$ of patients. Transient thrombocytopenia occurred in one patient that induced treatment discontinuation. Incidence of AEs were not related to the cumulative dose of isotretinoin or to patient gender. However, all AEs were transient. Xerosis and cheilitis were managed by topical emollient treatment. Isotretinoin-related depression and suicide risk is still debated. ${ }^{14}$ In our study no psychiatric AEs were observed, as well as clinical signs suggestive of inflammatory bowel disease or other rare side effects.

\section{4 | DISCUSSION}

This observational study confirmed the high efficacy of isotretinoin systemic treatment for moderate to severe acne with rapidly clinical and life's quality improvement. Generally, one cycle of therapy was sufficient, with a low risk of recurrence.

Although acne was more often severe in males compared with females at baseline, isotretinoin efficacy was not different in gender groups. It has been previously reported that male patients with acne are more frequently prescribed isotretinoin than females (OR: 2.44; 95\% Cl: 2.01-2.95). ${ }^{15}$ In agreement with these data, 59\% of our patients were males; in addition, patients were more prone to severe acne than females in our series.

Common or very common AEs, according to drug label, were recorded. None were unexpected according to the product sheet, and all $A E s$ were managed easily. ${ }^{1-3}$ Xerosis was the most frequent $A E$ and it was successfully treated with emollients.

Although the cumulative dosage in all subjects was lower than dosage from data sheet, patients who underwent the highest cumulative doses showed better clinical and quality of life response, with a reduced risk of recurrence. These results were obtained without an increased risk of $\mathrm{AEs}$, suggesting that adequate dosage must be used to maximize treatment effectiveness.

Besides the well known efficacy and safety of systemic isotretinoin in severe acne treatment, this study highlights that it could be used also in treatment of moderate acne with excellent results: higher effectiveness, less recurrence risk with only few transient AEs.

Thus, in the era of antibiotic resistance risk awareness, or in patients with known allergy reactions, as well as in those with high risk for scar formation, an early systemic isotretinoin treatment of moderate acne could be taken into consideration as a valid therapeutic approach. ${ }^{16}$

\section{ACKNOWLEDGMENTS}

No funding was secured for this study.

\section{CONFLICT OF INTEREST}

The authors declare no conflicts of interest.

\section{AUTHOR CONTRIBUTIONS}

C.P., N.S., and E.T. conceived of the present case report. E.T. wrote the manuscript. I.P., N.B., and A.M. contributed to the final version of the manuscript and supervised the project. V.B., P.M., A.M., S.M., and S.V. contributed to the manuscript revisions. All authors discussed the results and contributed to the final manuscript.

\section{DATA AVAILABILITY STATEMENT}

No data are available.

\section{ETHICS STATEMENT}

The authors assert that all procedures contributing to this work comply with the ethical standards of the relevant national and institutional committees on human experimentation and with the Helsinki Declaration of 1975 , as revised in 2008

\section{ORCID}

Ersilia Tolino (D) https://orcid.org/0000-0001-7861-9338 Nevena Skroza (iD https://orcid.org/0000-0003-4478-5404 Ilaria Proietti (D) https://orcid.org/0000-0003-3795-3190 Anna Marchesiello (D) https://orcid.org/0000-0002-5863-4829 Simone Michelini (DD https://orcid.org/0000-0002-3374-7384 Salvatore Volpe (D) https://orcid.org/0000-0002-6367-2344 Nicoletta Bernardini (D) https://orcid.org/0000-0002-6295-3574

\section{REFERENCES}

1. Das S, Reynolds RV. Recent advances in acne pathogenesis: implications for therapy. Am J Clin Dermatol. 2014;15(6):479-488.

2. Oge LK, Broussard A, Marshall MD. Acne vulgaris: diagnosis and treatment. Am Fam Physician. 2019;100(8):475-484.

3. Thiboutot DM, Dréno B, Abanmi A, et al. Practical management of acne for clinicians: an international consensus from the global Alliance to improve outcomes in acne. J Am Acad Dermatol. 2018;78(2 suppl 1):S11-S23.

4. Rademaker M. Isotretinoin: dose, duration and relapse. What does 30 years of usage tell us? Australas J Dermatol. 2013;54(3):157-162.

5. Rigopoulos D, Larios G, Katsambas AD. The role of isotretinoin in acne therapy: why not as first-line therapy? Facts and controversies. Clin Dermatol. 2010;28(1):24-30.

6. Veraldi S, Fabbrocini G, Cacciapuoti S, Masarà A. Terapia farmacologica. In Terapia dell'acne grave. Linee Guida SIDeMaST. https://www.pacinimedicina.it/wp-content/uploads/acne-gravesidemast-38709.pdf. Accessed February 2020).

7. Tkachenko E, Singer S, Sharma P, Barbieri J, Mostaghimi A. US Food and Drug Administration reports of pregnancy and pregnancy-related adverse events associated with isotretinoin. JAMA Dermatol. 2019; 155(10):1175-1179.

8. Droitcourt C, Poizeau F, Kerbrat S, et al. Isotretinoin and risk factors for suicide attempt: a population-based comprehensive case series and nested case-control study using 2010-2014 French health insurance data. J Eur Acad Dermatol Venereol. 2019;34(6):1293-1301. https://doi.org/10.1111/jdv.16005.

9. Droitcourt C, Nowak E, Rault C, et al. Risk of suicide attempt associated with isotretinoin: a nationwide cohort and nested case-timecontrol study. Int J Epidemiol. 2019;48(5):1623-1635. 
10. Singer S, Tkachenko E, Sharma P, Barbieri JS, Mostaghimi A. Psychiatric adverse events in patients taking isotretinoin as reported in a Food and Drug Administration database from 1997 to 2017. JAMA Dermatol. 2019;155(10):1162-1166.

11. Doshi A, Zaheer A, Stiller MJ. A comparison of current acne grading systems and proposal of a novel system. Int J Dermatol. 1997;36(6):416-418.

12. Hawthorne G, Richardson J, Osborne R. The assessment of quality of life (AQoL) instrument: a psychometric measure of health-related quality of life. Qual Life Res. 1999;8(3):209-224.

13. Martin AR, Lookingbill DP, Botek A, Light J, Thiboutot D, Girman CJ. Health-related quality of life among patients with facial acneassessment of a new acne-specific questionnaire. Clin Exp Dermatol. 2001;26(5):380-385.

14. Droitcourt C, Nowak E, Rault C, Happe A, Lw Natout B, Kerbrat S. Risk of suicide attempt associated with isotretinoin: a nationwide cohort and nested case-time-control study. Int J Epidemiol. 2019;48 (5):1623-1635.
15. Barbieri JS, Shin DB, Wang S, Margolis DJ, Takeshita J. Association of race/ethnicity and sex with differences in health care use and treatment for acne. JAMA Dermatol. 2020;156(3):312-319.

16. Tolino E, Skroza N, Proietti I, Bernardini N, Balduzzi V, Anzalone A. Post acne scarring: which factors are involved? J Ital Dermatol Venereol. 2019;154(4):154-156.

How to cite this article: Tolino E, Skroza N, Proietti I, et al. Efficacy and safety of systemic isotretinoin treatment for moderate to severe acne (insights from the real-life clinical setting). Dermatologic Therapy. 2020;33:e14392. https://doi. org/10.1111/dth.14392 